\title{
MESHLESS COLLOCATION METHODS APPLIED TO PROBLEMS WITH MATERIAL DISCONTINUITIES
}

\author{
ARTUR KROWIAK \\ Department of Applied Computer Science, Cracow University of Technology \\ Al. Jana Pawła II 37, 31-864 Kraków, Poland \\ artur.krowiak@pk.edu.pl
}

Key words: Material discontinuity, Interface problem, Mercer series, Stable RBF basis.

\begin{abstract}
The paper deals with the use of a kind of meshless method to solve the problem with material discontinuity on an interface. Such a problem is described by a differential equation with discontinuous coefficients. To solve the problem, the abovementioned method is associated with the subdomain approach that divides the whole domain into subdomains, in which the problem is continuous. To accurately address the analyzed problem, proper continuity conditions are imposed on the interface. The Gaussian kernel, which belongs to the family of infinitely smooth radial basis functions, is taken into consideration as the basis function for the method. It is known that this type of method can provide very high rate of convergence and high accuracy but it suffers from instability. To avoid the instability, some recent advances in kernel methods, based on Mercer's theorem, are involved in the present paper. The usefulness of the approaches are shown by benchmark problems described by ordinary as well as partial differential equations.
\end{abstract}

\section{INTRODUCTION}

Due to some features, the kernel functions are often used as bases for many approximation problems. Using such an approximation, which is associated with the collocation procedure, it is easy to discretize differential equations defined on irregular domains and in higher dimensions. The kernel methods have been recently applied in many scientific and engineering problems [1, 2]. In most cases, the radial basis functions (RBFs) play the role of the kernels. In many papers (e.g. [3]) devoted to the methods based on the global RBFs, it is pointed out that these methods are able to yield high accuracy, in some cases exponential one, but this accuracy is achievable for near flat RBFs. The flatness of these functions is controlled by a constant called the shape parameter. Unfortunately, the values of this parameter that theoretically should lead to high accuracy make the system matrix ill-conditioned and the problem difficult to solve.

To overcome the problem, several approaches have been developed [4-6]. Most of them are based on searching for a proper value of the shape parameter as a trade-off between accuracy and stability. In other approaches an attempt is made to find an alternate, stable basis spanned over the space created by the RBF kernels.

In $[7,8]$ the new basis is obtained by QR or SVD factorization of kernel matrix. In [9], another interesting approach is presented. It differs from the mentioned ones by the fact that the 
kernel matrix, which is prone to be ill-conditioned, is not formed. In order to obtain a stable basis appropriate factorization of the kernel matrix is done on the base of its approximation following from Mercer's theorem. Thus far, this method has been successfully applied in some approximation problems and for solving differential equations $[10,11]$.

In [12], the stable basis was used to form the sought solution for ordinary differential equations with discontinuous coefficients. This mathematical model may often describe some engineering problems, when the analysed element is composed of different materials linked by interfaces. Using a collocation procedure associated with subdomain approach the stable solution of high accuracy can be achieved, as it is shown in [12]. The present paper is an extension of this work. In the present paper, three ways for the derivation of the alternate basis, starting from Mercer's theorem, are examined. These approaches differ from each other in assuming different terms in the Mercer series in relation to the number of nodes. They are described in detail in [1] and in this paper they are involved in the kernel-based method to solve the problem with material discontinuity on the interface.

The paper is organized as follows: in section 2 a general description of the kernel-based method is presented, in section 3 three ways for obtaining the alternate, stable basis are shown, in section 4 the treatment of the discontinuity is explained and details of the discretization process are presented. Some results of the numerical tests are shown in section 5 and concluding remarks are finally drawn.

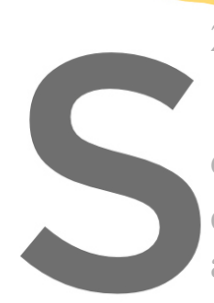

2 KERNEL-BASED METHODS

The kernel function is understood as a real-valued function of

dimensional space,

conveniently construct data dependent basis, which is a

approximation problem

defined

nsions $[12]$

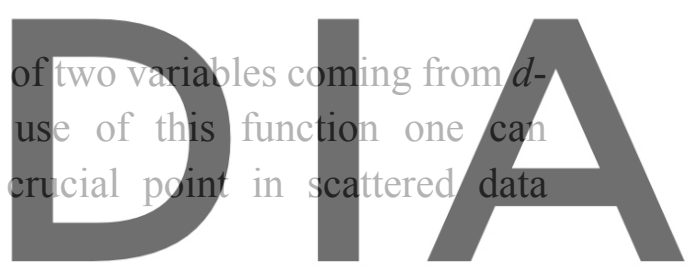

Assuming a set of data points $\left\{\left(\mathbf{x}_{i}, y_{i}\right)\right\}_{i=1}^{N}$ with $\mathbf{x}_{i} \subset \Omega \in \mathbb{R}^{d}, y_{i} \in \mathbb{R}$, the kernel basis of the

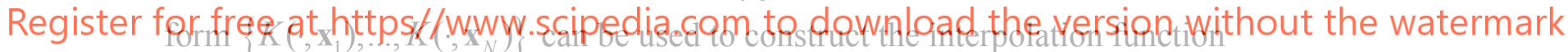

$$
s(\mathbf{x})=\sum_{j=1}^{N} c_{j} K\left(\mathbf{x}, \mathbf{x}_{j}\right)=\mathbf{k}(\mathbf{x})^{T} \mathbf{c}
$$

where $\mathbf{k}(\mathbf{x})^{T}=\left[\begin{array}{lll}K\left(\mathbf{x}, \mathbf{x}_{1}\right) & \cdots & K\left(\mathbf{x}, \mathbf{x}_{N}\right)\end{array}\right]$ is a vector of the base functions and $\mathbf{c}$ is a vector of the interpolation coefficients, obtained after imposing interpolation conditions $s\left(\mathbf{x}_{i}\right)=y_{i}, i=$ $1, \ldots, N$. The latter, written in matrix notation, assumes the form

$$
\mathbf{K c}=\mathbf{y}
$$

where $\mathbf{y}$ is a vector of the function values at the respective nodes and $\mathbf{K}$ is so-called kernel matrix that has the following form

$$
\mathbf{K}=\left[\begin{array}{c}
\mathbf{k}\left(\mathbf{x}_{1}\right)^{T} \\
\vdots \\
\mathbf{k}\left(\mathbf{x}_{N}\right)^{T}
\end{array}\right]
$$


For the positive-definite kernel functions, the above matrix is also positive-definite and therefore invertible. It means that the interpolation coefficients can be obtained from Eq. (2) and the interpolation function (1) is determined.

When solving a differential equation it is not needed to invert the kernel matrix. Let us consider a boundary value problem in a general form as

$$
L u=f \text { in } \Omega, \quad B u=g \text { on } \partial \Omega
$$

where $L$ and $B$ denote linear differential operators imposed on the sought function $u$ in the domain $\Omega$ and on the boundary $\partial \Omega$, respectively and $f, g$ are known functions.

After discretizing the domain as well as the boundary using scatter nodes $\mathbf{x}_{i}$, we can construct the sought solution for the differential problem using the kernel basis as in Eq. (1), i.e.

$$
u_{h}(\mathbf{x})=\mathbf{k}(\mathbf{x})^{T} \mathbf{c}
$$

After introducing the sought solution to Eq. (4) and applying the collocation procedure, one obtains

$$
\mathbf{K}_{L B} \cdot \mathbf{c}=\mathbf{F}
$$

The structures of system matrix $\mathbf{K}_{L B}$ and vector $\mathbf{F}$ are as follows
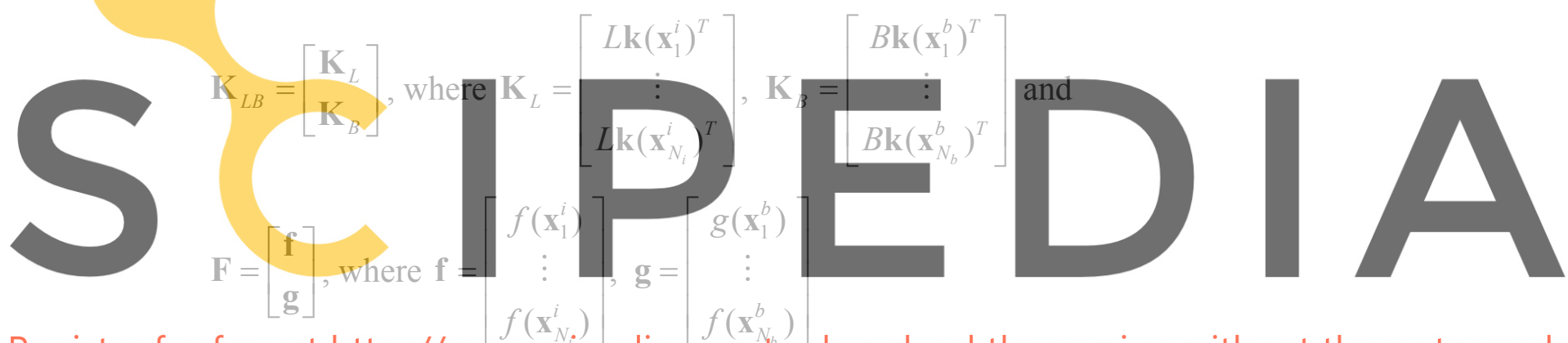

Register for free at https//www.scipedia.com to download the version without the watermark

In the above objects, there is a distinction between interior nodes $\mathbf{x}_{i}^{i}, j=1, \ldots, N_{i}$ and boundary nodes $\mathbf{x}_{j}^{b}, j=1, \ldots, N_{b}$. Note that the total number of nodes is $N=N_{i}+N_{b}$.

If only the system matrix of Eq. (6) is not singular, the interpolation coefficients can be computed and the approximate solution in the form of Eq. (5) is determined. As one can see, the procedure of the kernel-based method applied to the solution of differential equations is easy. Moreover, the solution is obtained in the analytic form, which can be viewed as another advantage of the method. As the kernel function, the RBFs are willingly used.

The main drawback of the method is high ill-conditioning of the system (Eq.(6)). When using RBFs, this ill-conditioning can be controlled by properly determined shape parameter but then the theoretically estimated high accuracy of the method is not achievable.

In order to maintain the possibility of achieving spectral accuracy, another way to overcome the instability is applied in the present paper.

\section{DERIVATION OF THE STABLE BASIS}

According to Mercer's theorem, every positive definite kernel can be represented by an 
infinite series composed of eigenvalues $\lambda_{n}$ and eigenfunctions $\varphi_{n}$ of associated HilbertSchmidt eigenvalue problem [9]. This representation can be put as follows

$$
K(\mathbf{x}, \mathbf{z})=\sum_{n=1}^{\infty} \lambda_{n} \varphi_{n}(\mathbf{x}) \varphi_{n}(\mathbf{z})
$$

Since the Gaussian RBF $\mathrm{e}^{-\varepsilon^{2}\|\mathbf{x}-\boldsymbol{z}\|^{2}}$ is a positive definite kernel it can be presented in such a way. Generally, if one operates in $d$-dimensional spaces, $d$-dimensional eigenvalues and eigenfunctions for the Gaussian kernel follow immediately from the univariate ones via tensor product form of this kernel. The details of this representation can be found in $[1,12]$.

To take advantage of Mercer's theorem in numerical computation, the series has to be truncated. The number of terms, which is taken into account, in relation to number of nodes leads to three general approaches for the derivation of an alternate basis. These approaches are described in detail in [1] but for the consistency they are presented herein in a brief form.

\subsection{Direct approach}

Assuming that we use the truncated series with the number of terms matching the number of $\operatorname{nodes} N$, the approximation of the kernel takes the following form

$$
K\left(\mathbf{x}, \mathbf{x}_{j}\right)=\sum_{n=1}^{N} \lambda_{n} \varphi_{n}(\mathbf{x}) \varphi_{n}\left(\mathbf{x}_{j}\right)=\phi(\mathbf{x})^{T} \Lambda \phi\left(\mathbf{x}_{j}\right)
$$
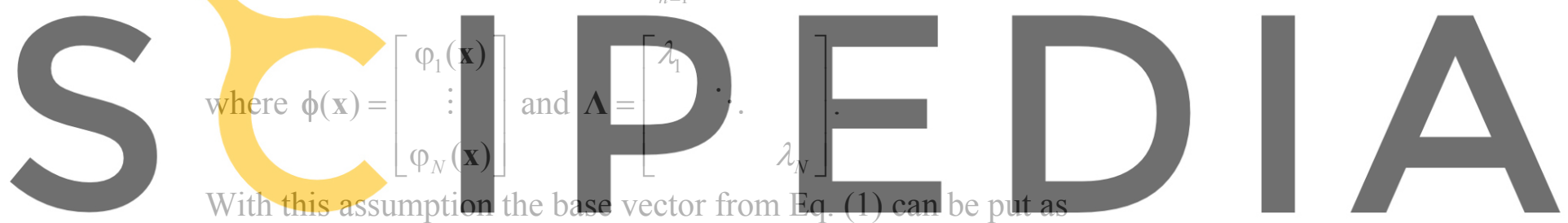

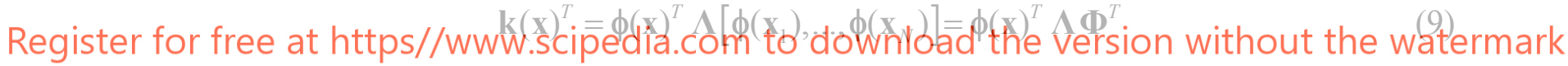

and the kernel matrix is as follows

$$
\mathbf{K}=\boldsymbol{\Phi} \Lambda \boldsymbol{\Phi}^{T}
$$

where $\boldsymbol{\Phi}=\left[\begin{array}{c}\phi\left(\mathbf{x}_{1}\right)^{T} \\ \vdots \\ \phi\left(\mathbf{x}_{N}\right)^{T}\end{array}\right]$

It is worth recalling that if $\mathbf{x} \in \mathbb{R}^{d}$ the eigenvalues and eigenfunctions contained in the above equations are easily obtained from the univariate ones by appropriate multiplication.

Now, the interpolant given by Eq. (1) can be presented with the aid of Eqs. (2), (9) and (10) in the following form

$$
S(\mathbf{x})=\underbrace{\phi(\mathbf{x})^{T} \Lambda \mathbf{\Phi}^{T}}_{\mathbf{k}(\mathbf{x})^{T}} \underbrace{\Phi^{-T} \Lambda^{-1} \mathbf{\Phi}^{-1}}_{\mathbf{K}^{-1}} \mathbf{y}=\phi(\mathbf{x})^{T} \mathbf{\Phi}^{-1} \mathbf{y}
$$

In Eq. (11) $\phi^{T}(\mathbf{x}) \boldsymbol{\Phi}^{-1}$ are the cardinal basis functions. From another point of view, the 
interpolant (11) can be put as

$$
s(\mathbf{x})=\phi(\mathbf{x})^{T} \mathbf{b}
$$

where $\mathbf{b}=\boldsymbol{\Phi}^{-1} \mathbf{y}$. In this notation the vector of eigenfunctions is the vector of the base functions.

As it is pointed out in [9], the ill-conditioning of the kernel matrix is associated with the eigenvalues, i.e. with matrix $\Lambda$. Now this ill-conditioning is algebraically removed, when forming the interpolant (see Eq. (11)). In the view of Eq. (12) the vector of eigenfunctions can be considered as a vector of the stable basis for the kernel-based methods.

\subsection{Low-rank approximation}

The number of terms $M$ in the Mercer series can be less than the number of nodes, i.e. $M<$ $N$. Then, the kernel approximation takes the following form

$$
K\left(\mathbb{x}, \mathbf{x}_{j}\right)=\sum_{n=1}^{M} \lambda_{n} \varphi_{n}(\mathbb{x}) \varphi_{n}\left(\mathbb{x}_{j}\right)=\phi(\mathbf{x})^{T} \Lambda \phi\left(\mathbf{x}_{j}\right)
$$

where respective objects $\phi(\mathbf{x})$ and $\Lambda$ have the structure similar as in Eq. (8) but possess fewer number of elements - up to $M$. In this case the kernel matrix $\mathbf{K}$ is numerically low-rank and its inversion in the context of Eq. (11) is not available.

Now one can write the interpolant as
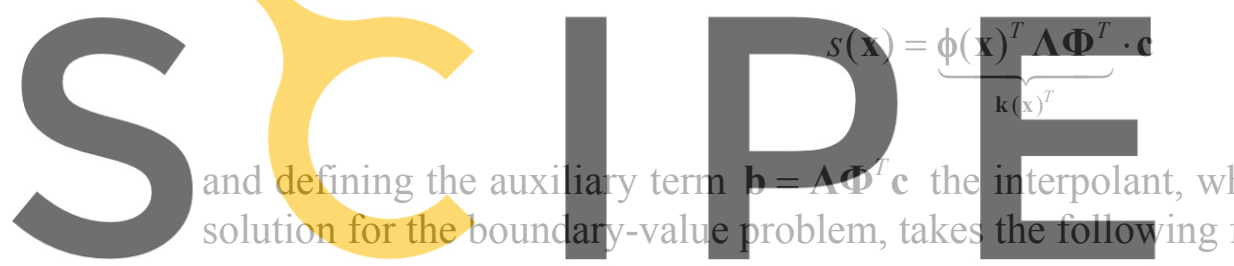

$$
u_{h}(\mathbf{x})=s(\mathbf{x})=\phi(\mathbf{x})^{T} \mathbf{b}
$$

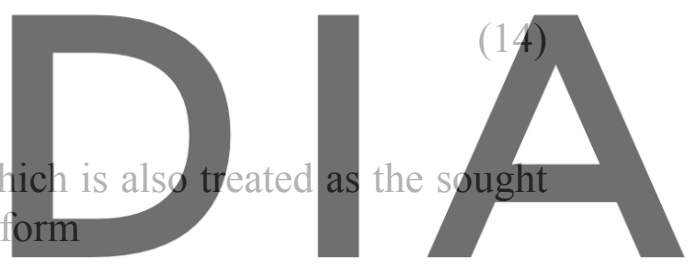

(15)

Register for free at https//www.scipedia.com to download the version without the watermark

In this case, the vector of the eigenfunctions becomes also the base vector for the approximation of the sought solution of the considered problem. But now the collocation procedure does not lead to the square system of equations, since the number of elements in the base vector is lower than the number of nodes. The discretized system of a boundary-value problem, corresponding to Eq. (6), has the following form

$$
\underbrace{\boldsymbol{\Phi}_{L B}}_{N \times M} \cdot \mathbf{b}=\mathbf{F}
$$

and can be solved e.g. in the least squares sense, finding such vector $\mathbf{b}$ that minimizes $\left\|\boldsymbol{\Phi}_{L B} \cdot \mathbf{b}-\mathbf{F}\right\|_{2}^{2}$. The solution can be put as

$$
\mathbf{b}=\left(\boldsymbol{\Phi}_{L B}^{T} \boldsymbol{\Phi}_{L B}\right)^{-1} \boldsymbol{\Phi}_{L B}^{T} \mathbf{F}
$$

In Eqs. (16) and (17) $\boldsymbol{\Phi}_{L B}$ is the system matrix, similar to $\mathbf{K}_{L B}$ in Eq. (6), where the differential operators are imposed on $\phi(\mathbf{x})^{T}$ base vector. 


\subsection{Hilbert-Schmidt SVD}

If more terms are taken in the Mercer series than the number of nodes $(M>N)$, the base vector, corresponding to Eq. (9), can be written using block matrices, i.e.

$$
\boldsymbol{\Phi}=\left[\begin{array}{ll}
\boldsymbol{\Phi}_{1} & \boldsymbol{\Phi}_{2}
\end{array}\right], \quad \boldsymbol{\Lambda}=\left[\begin{array}{cc}
\boldsymbol{\Lambda}_{1} & \mathbf{0} \\
\mathbf{0} & \boldsymbol{\Lambda}_{2}
\end{array}\right]
$$

where $\boldsymbol{\Phi}_{1}, \boldsymbol{\Lambda}_{1} \in \mathbb{R}^{N \times N}, \boldsymbol{\Phi}_{2} \in \mathbb{R}^{N \times(M-N)}, \boldsymbol{\Lambda}_{2} \in \mathbb{R}^{(M-N) \times(M-N)}$.

With above notation the base vector takes the following form

$$
\mathbf{k}(\mathbf{x})^{T}=\boldsymbol{\phi}(\mathbf{x})^{T} \boldsymbol{\Lambda} \boldsymbol{\Phi}^{T}=\boldsymbol{\phi}(\mathbf{x})^{T}\left[\begin{array}{cc}
\boldsymbol{\Lambda}_{1} & \mathbf{0} \\
\mathbf{0} & \boldsymbol{\Lambda}_{2}
\end{array}\right]\left[\begin{array}{l}
\boldsymbol{\Phi}_{1}^{T} \\
\boldsymbol{\Phi}_{2}^{T}
\end{array}\right]
$$

After simple algebraic manipulations, Eq. (19) can be expressed as

$$
\mathbf{k}(\mathbf{x})^{T}=\phi(\mathbb{x})^{T}\left[\begin{array}{c}
\mathbb{I}_{N} \\
\Lambda_{2} \Phi_{2}^{T} \Phi_{1}^{-T} \Lambda_{1}^{-1}
\end{array}\right] \Lambda_{1} \Phi_{1}^{T}=\psi(\mathbb{x})^{T} \Lambda_{1} \Phi_{1}^{T}
$$

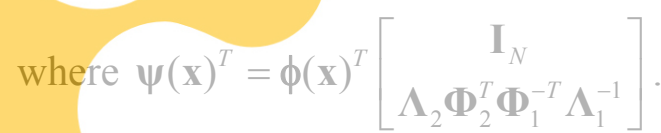

With the above notation the kernel matrix, corresponding to Eq. (10), takes the following

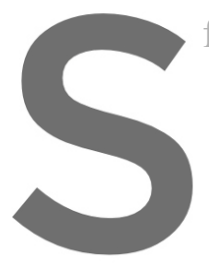
form
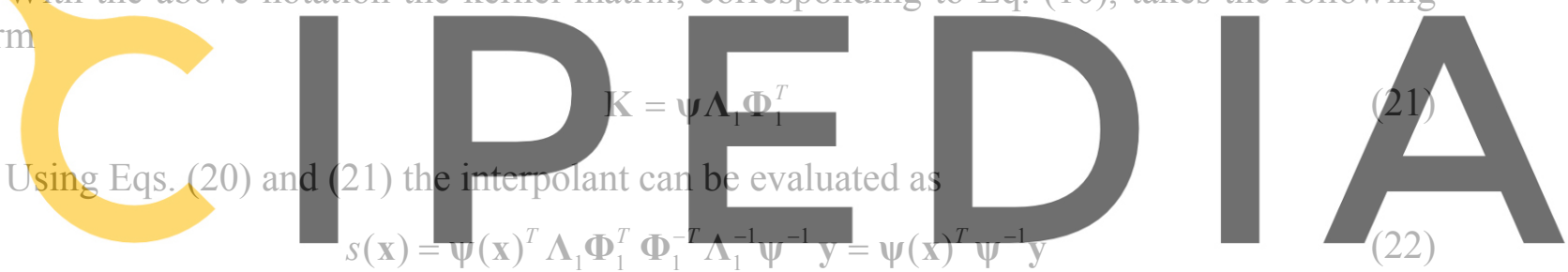

Register for free at https//www.scipedia.com to download the version without the watermark

By defining a new set of coefficients $\mathbf{b}=\psi^{-1} \mathbf{y} \mathrm{Eq}$. (22) assumes the following form

$$
s(\mathbf{x})=\psi(\mathbf{x})^{T} \mathbf{b}
$$

Although the derived basis $\psi(\mathbf{x})^{T}$ contains the matrices $\Lambda_{1}, \Lambda_{2}$, as much as possible the illconditioning that comes from the matrix $\Lambda$ is isolated and removed, as shown by Eq. (22). Therefore $\psi(\mathbf{x})^{T}$ can be considered as an alternate stable basis for the kernel-based method.

The name of this approach follows from the fact that the approximation of the kernel matrix (Eq. (21)) has structural similarity to the traditional SVD. It is worth noticing that the derivation of this basis is computationally much less efficient comparing to those from sections 3.1 and 3.2 .

\section{SOLUTION OF THE INTERFACE PROBLEM}

Let us take into consideration the boundary-value problem introduced by Eq. (4), where differential operator $L$ possesses discontinuous coefficients on the interface, e.g. due to the different materials used to build the analysed element. Let us assume that the domain of this heterogeneous problem can be divided into several subdomains, in the way that in each of them 
the problem is homogeneous. Such a general case composed of $n$ subdomains is presented in Fig. 1.

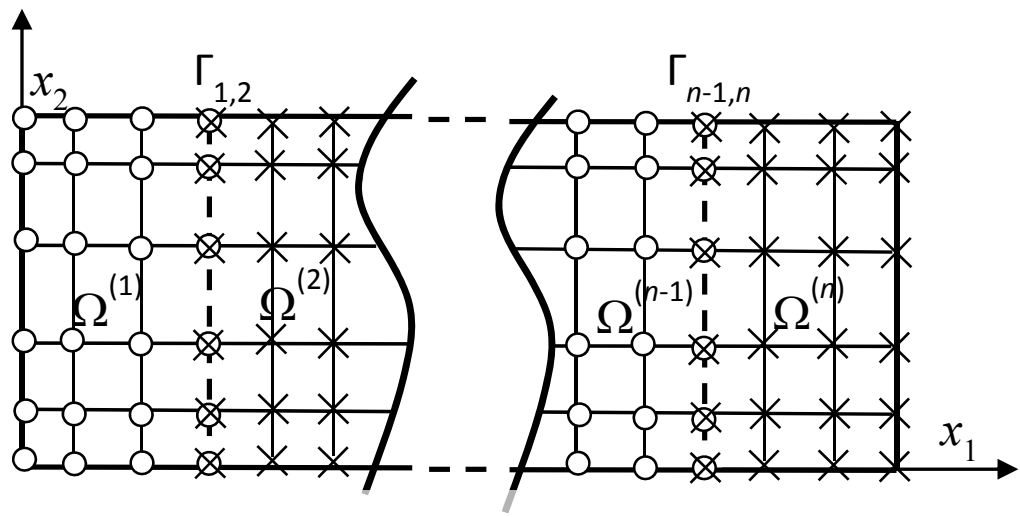

Figure 1: Domain of the problem with node discretization

\section{The original problem can be considered in each subdomain separately as}

$$
\begin{aligned}
& L^{(1)} u^{(1)}=f^{(1)} \text { in } \Omega^{(1)}, \quad B^{(1)} u^{(1)}=g^{(1)} \text { on } \partial \Omega^{(1)} \\
& L^{(2)} u^{(2)}=f^{(2)} \text { in } \Omega^{(2)}, \quad B^{(2)} u^{(2)}=g^{(2)} \text { on } \partial \Omega^{(2)}
\end{aligned}
$$
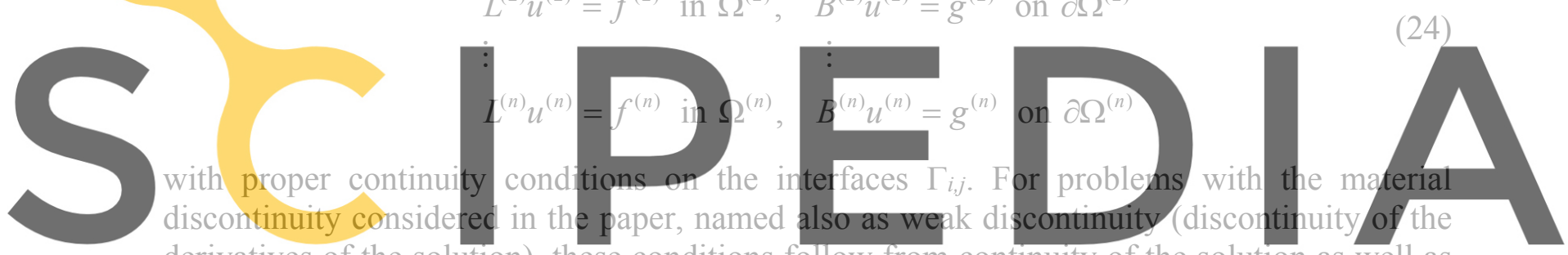

derivatives of the solution), these conditions follow from continuity of the solution as well as

Register forfreety of the traction or flux, dependently on the physical problem considered. They assume

$$
\begin{aligned}
& u^{(1)}(\mathbf{x})=u^{(2)}(\mathbf{x}), \quad T^{(1)} u^{(1)}(\mathbf{x})=T^{(2)} u^{(2)}(\mathbf{x}) \quad \text { for } \quad \mathbf{x} \in \Gamma_{1,2} \\
& \vdots \\
& \vdots \\
& u^{(n-1)}(\mathbf{x})=u^{(n)}(\mathbf{x}), \quad T^{(n-1)} u^{(n-1)}(\mathbf{x})=T^{(n)} u^{(n)}(\mathbf{x}) \text { for } \mathbf{x} \in \Gamma_{n-1, n}
\end{aligned}
$$

where $T$ denotes respective differential operator.

To form the interpolation function for the sought solution of the problem, the stable basis, derived in previous section, is used. It can be put as

$$
u(\mathbf{x})= \begin{cases}u^{(1)}(\mathbf{x})=\phi^{(1)}(\mathbf{x})^{T} \mathbf{b}^{(1)}, & \mathbf{x} \in \bar{\Omega}^{(1)} \\ u^{(2)}(\mathbf{x})=\phi^{(2)}(\mathbf{x})^{T} \mathbf{b}^{(2)}, & \mathbf{x} \in \bar{\Omega}^{(2)} \\ \vdots & \\ u^{(n)}(\mathbf{x})=\phi^{(n)}(\mathbf{x})^{T} \mathbf{b}^{(n)}, & \mathbf{x} \in \bar{\Omega}^{(n)}\end{cases}
$$

Where $\bar{\Omega}^{(i)}=\Omega^{(i)} \cup \partial \Omega^{(i)} \cup \Gamma_{i, j}$ represents the closed domain. If the HS-SVD basis is used 
$\phi^{(i)}(\mathbf{x})^{T}$ is replaced by $\psi^{(i)}(\mathbf{x})^{T}$. The number of the eigenfunctions in vector $\phi^{(i)}(\mathbf{x})$, when the basis from section 3.1 is taken, corresponds with the number of nodes imposed on $\bar{\Omega}^{(i)}$ domain, i.e. $N^{(i)}$. The same situation takes place, when the HS-SVD basis is used - the number of elements of $\psi^{(i)}(\mathbf{x})^{T}$ corresponds to the number of nodes in the $i$ th subdomain. But if one uses low rank approximation (section 3.2), the number of eigenfunctions in vector $\boldsymbol{\phi}^{(i)}(\mathbf{x})$ is less than the number of nodes in the respective subdomain. As Fig. 1 shows, on the interface, at the same location there are two nodes that come from adjacent subdomains, allowing for two continuity conditions to be collocated at this location, as Eq. (25) suggests.

Substituting Eq. (26) into Eqs. (24) and (25), then collocating respective equations at each node in the domain, on the boundary and on the interface one obtains a discrete system of equations, which can be put in the following matrix notation

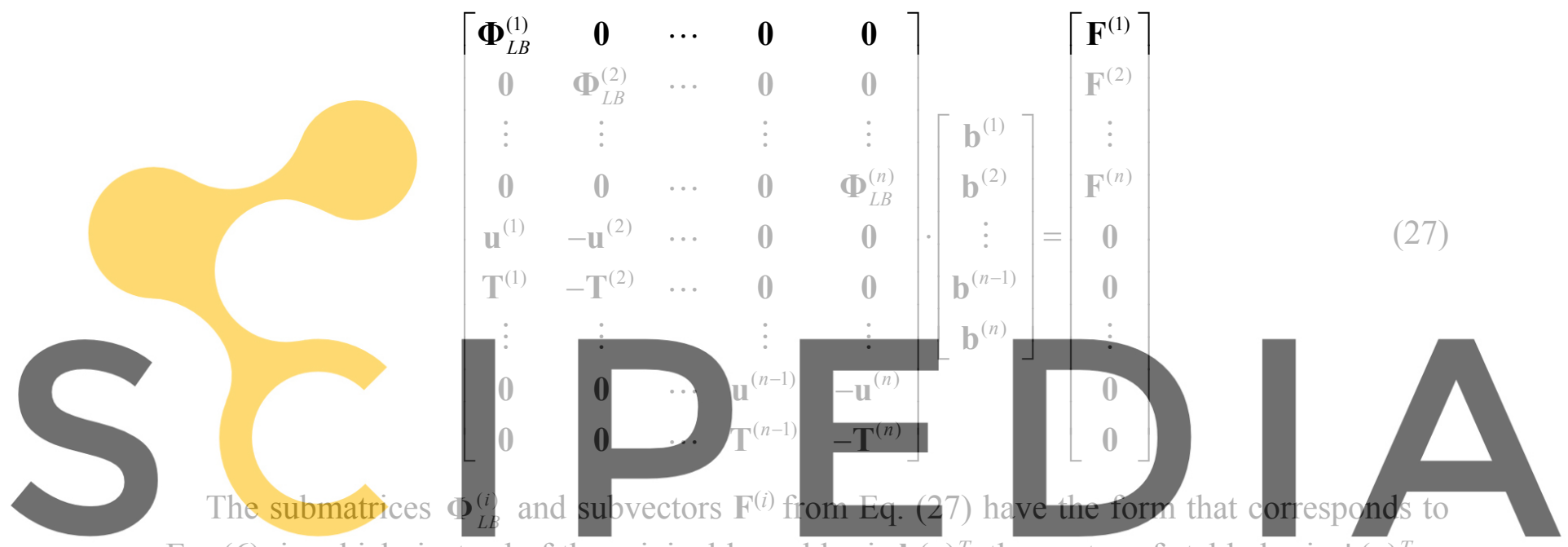

Eq. (6), in which, instead of the original kernel basis $\mathbf{k}(\mathbf{x})^{T}$, the vector of stable basis $\phi(\mathbf{x})^{T}$ or

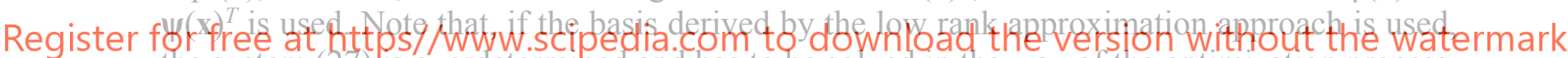
the system (27) is overdetermined and has to be solved in the way of the optimization process, as it is described in section 3.2. The submatrices $\mathbf{u}^{(i)}$ and $T^{(i)}$ are the result of the collocation of the interface conditions (25) at the interface nodes and have the following structure

$$
\mathbf{u}^{(i)}=\left[\begin{array}{c}
\phi^{(i)}\left(\mathbf{x}_{1}^{\Gamma_{i, j}}\right)^{T} \\
\vdots \\
\phi^{(i)}\left(\mathbf{x}_{N_{\Gamma}}^{\Gamma_{i, j}}\right)^{T}
\end{array}\right], \quad \mathbf{T}^{(i)}=\left[\begin{array}{c}
T^{(i)} \phi^{(i)}\left(\mathbf{x}_{1}^{\Gamma_{i, j}}\right)^{T} \\
\vdots \\
T^{(i)} \phi^{(i)}\left(\mathbf{x}_{N_{\Gamma}, j}^{\Gamma_{i, j}}\right)^{T}
\end{array}\right]
$$

Once the system (27) is solved for coefficients $\mathbf{b}^{(i)}$, the approximate solution given by Eq. (26) is determined in each subdomain.

\section{NUMERICAL RESULTS}

The accuracy and stability of the method is examined by ordinary and partial differential equations. The first test problem describes a diffusion in a cylindrical annulus that possesses two interfaces, where the diffusion parameter has some jumps. Due to the axisymmetry, the analysed problem can be described by the following equation 


$$
D \frac{d^{2} u}{d r^{2}}+\frac{D}{r} \frac{d u}{d r}=0, \quad u\left(r_{1}\right)=1, u\left(r_{2}\right)=2, D=\left\{\begin{array}{c}
1 \text { for } r \in[0.025,0.25] \\
0.1 \text { for } r \in(0.25,0.5] \\
0.01 \text { for } r \in(0.5,1]
\end{array}\right.
$$

and viewed as a steady, one-dimensional convection-diffusion problem.

Since $D$ coefficient has different values in adjacent subdomains, one can expect non-smooth solution with discontinuous derivative. The solution presented in Fig. 2 was obtained using the basis from the section 3.1 with $N^{(1)}=6, N^{(2)}=6, N^{(3)}=11$ nodes. The nodes were uniformly distributed in each subdomain.

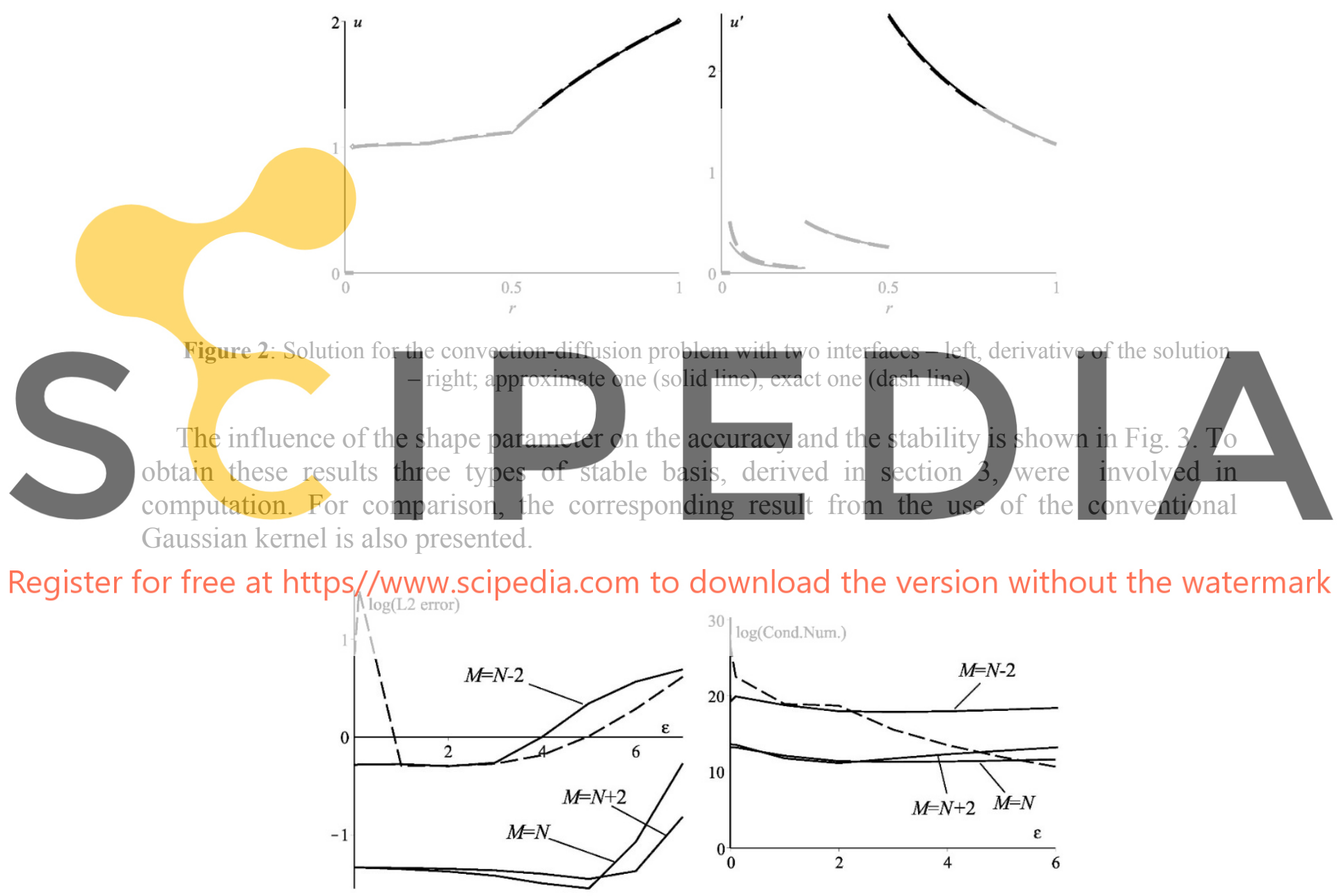

Figure 3: Results obtained for the problem with two interfaces. Error in $L_{2}$ norm vs. shape parameter - left, condition number vs. shape parameter - right; stable basis (solid line), RBF with Gaussian kernel (dash line)

Figure 3 shows that the most accurate solution is determined when the square system is generated using the base from section $3.1\left(M^{(i)}=N^{(i)}\right)$ and HS-SVD $\left(M^{(i)}>N^{(i)}\right)$. These solutions converge to each other for $\varepsilon \rightarrow 0$. The conditioning of these systems is almost independent of the shape parameter, oppositely to the system generated by the use of the standard Gaussian kernel (dash line). In this case, a highly ill-conditioned system makes it impossible to achieve 
high accuracy for small value of $\varepsilon$, i.e. for near flat Gaussian function. It should be noted that the condition number for the low-rank approximation $\left(M^{(i)}<N^{(i)}\right)$, which leads to overdetermined system, is computed for the system that follows from the least squares technique (corresponding to Eq. (17)). Figure 4 shows the absolute error distribution within whole domain. One can note that there is not a significant loss of accuracy at the interfaces denoted in the figure by dash lines. The convergence test, presented also in Fig. 4, suggests that the method exhibits exponential convergence. The results displayed in Fig. 4 were obtained with $M^{(i)}=N^{(i)}$.
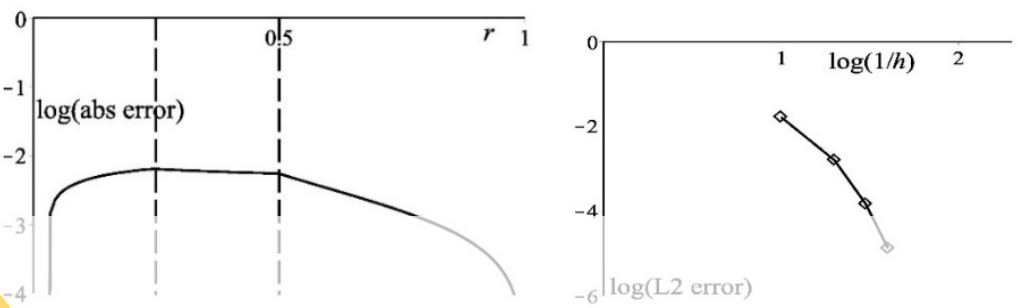

Figure 4: Absolute error distribution - left and convergence rate- right

The second test problem is described by the elliptic equation of the following form $-\nabla(k(\mathbf{x}) \nabla u)=f(\mathbf{x}), \quad \mathbf{x}=\left(x_{1}, x_{2}\right) \in \Omega \subset \mathbb{R}^{2}$,

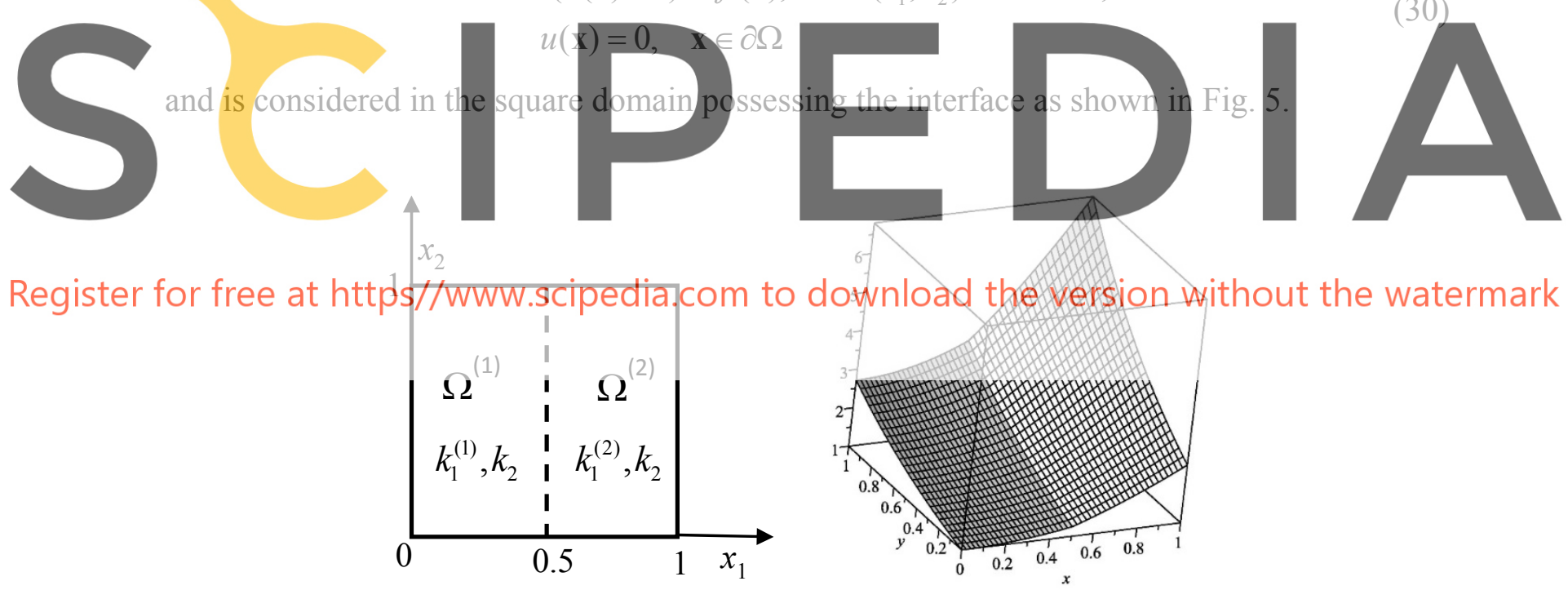

Figure 5: The domain of 2D elliptic interface problem - left and the approximate solution - right

It is assumed that the material is orthotropic i.e. tensor $\mathrm{k}(\mathbf{x})$ has two nonzero elements $k(\mathbf{x})=\left[k_{1}(\mathbf{x}), k_{2}(\mathbf{x})\right]$ that denote the conductivity in two perpendicular directions. With this assumption Eq. (30) takes the following form

$$
-\frac{\partial}{\partial x_{1}}\left(k_{1}(\mathbf{x}) \frac{\partial u}{\partial x_{1}}\right)-\frac{\partial}{\partial x_{2}}\left(k_{2}(\mathbf{x}) \frac{\partial u}{\partial x_{2}}\right)=f(\mathbf{x})
$$


Moreover, it is assumed that coefficient $k_{1}(\mathbf{x})$ has discontinuity on the interface, as it is shown in Fig. 5.

The solution obtained by the use of the basis from section 3.1 , where $M^{(i)}=N^{(i)}$, is shown in Fig. 5. To obtain the solution, $N^{(1)}=N^{(2)}=90$ nodes were scattered over each subdomain. In the computation the following values of the coefficients were chosen: $k_{1}^{(1)}=2, k_{1}^{(2)}=1, k_{2}=1$.

To examine the accuracy of the method as well as the conditioning of the system and their dependence on the shape parameter, several numerical tests have been carried out. The results are presented in Fig. 6.
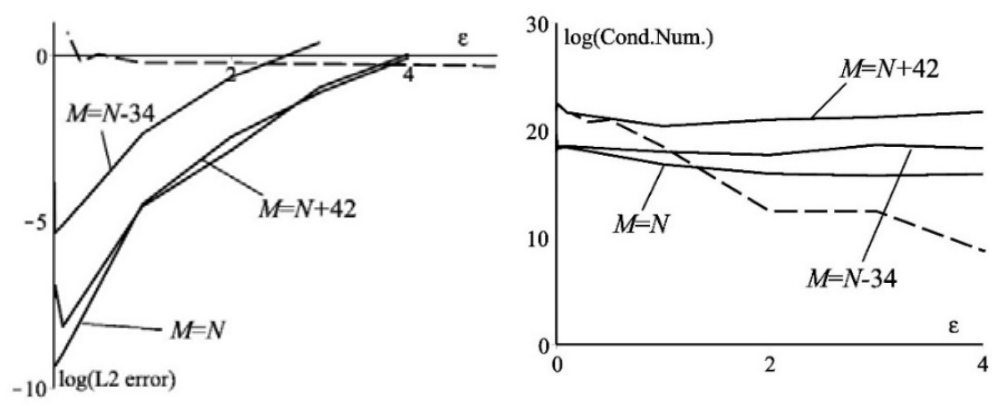

Figure 6: Error in $L_{2}$ norm vs. shape parameter - left, condition number vs. shape parameter - right; stable basis (solid line), RBF with Gaussian kernel (dash line)

As in the previous example the direct approach $\left(M^{(i)}=N^{(i)}\right)$ and HS-SVD $\left(M^{(i)}>N^{(i)}\right)$ basis provide the most accurate results. The conditioning of the systems generated by the use of the stable basis is almost independent of the shape parameter. Therefore, instead of employing special algorithms to search for the optimal value of $\varepsilon$, one can take very small this value and expect the most accurate results that go with theoretical findings.

\section{CONCLUSION}

In the present paper, the kernel-based method is applied to solve the interface problems arising from material discontinuity. To this end, the method was associated with subdomain approach, which using appropriate interface conditions allows us to solve the problem conveniently. In order to overcome the drawbacks with ill-conditioning of the Gaussian kernel, some approaches for derivation of stable basis were employed. All the approaches take advantage of the approximation of positive definite kernel by the series of its eigenvalues and eigenfunctions. After some numerical tests carried out in the work, several remarks can be drawn:

- All the approaches give results that coincide with theoretical findings, i.e. that the best accuracy can be achieved for near flat RBF kernels (when the shape parameter approaches zero).

- Among these approaches, the direct one turns out to be the most reasonable (when the number of terms $M$ taken in the Mercer series equals the number of nodes $N$ ). HS-SVD basis, derived in the case when $M>N$, leads to similar accuracy as the direct approach but the computational efficiency at the stage of the base derivation is a serious drawback. 
The method presented in this article may also be useful in similar issues, e.g. in the analysis of 2D [13] and 3D images [14] obtained from optical observations and microtomography, where both the process of data analysis and material structure reconstruction at the level of mathematical formalism is close to or even isomorphic to material body analysis.

\section{REFERENCES}

[1] Fasshauer, G.E. and McCourt, M.J. Kernel-based Approximation Methods using Matlab. World Scientific, (2015).

[2] Buhmann, M.D. Radial basis functions: Theory and Implementations. Cambridge University Press, (2003).

[3] Schaback, R. Error estimates and condition numbers for radial basis function interpolation. Adv. Comput. Math. (1995) 3:251-264.

[4] Rippa, S. An algorithm for selecting a good value for the parameter $c$ in radial basis function interpolation. Adv. Comput. Math. (1999) 11:193-210.

[5] Tsai, C.H. et al. The golden section search algorithm for finding a good shape parameter for meshless collocation methods. Eng. Anal. Bound. Elem. (2010) 34:738-746.

[6] Krowiak, A. Domain-type RBF Collocation Methods for Biharmonic Problems. Int. J. Comp. Meth.-Sing. (2018) 15:1850078-1-1850078-20.

[7] De Marchi, S. and Santin, G. A new stable basis for radial basis function interpolation. $J$. Comput. Appl. Math. (2013) 253: 1-13.

[8] Pazouki, M. and Schaback, R. Bases for kernel-based spaces. J. Comput. Appl. Math. (2011) 236:575-588.

[9] Fasshauer, G.E. and McCourt, M.J. Stable evaluation of Gaussian radial basis function interpolants. SIAM J. Sci. Comput. (2012) 34:A737-A762.

[10] Rashidinia, J. et al. A stable method for the evaluation of Gaussian radial basis function solution of interpolation and collocation problems. Comput. Math. Appl. (2016) 72:178-193.

[11] Rashidinia, J. et al. A stable Gaussian radial basis function method for solving nonlinear unsteady convection-diffusion-reaction equations. Comput. Math. Appl. (2018) 75:18311850.

[12] Krowiak, A. and Podgórski, J. Stable evaluation of Gaussian kernel applied to interface problems. Proceedings of the 4th International Conference on Numerical and Symbolic Computation - Developments and Applications, Symcomp 2019, Porto

[13] Radek, N., Szczotok, A., Gądek-Moszczak, A., Dwornicka, R., Broncek, J. and Pietraszek J. The impact of laser processing parameters on the properties of electro-spark deposited coatings. Arch. Metall. Mater. (2018) 63:809-816.

[14] Gądek-Moszczak, A. and Żmudka, S. Description of 3D microstructure of the composites with polypropylene (PP) matrix and tuf particles fillers. Solid State Phenomena (2013) 197:186-191. 